\title{
Peroxiredoxins are involved in two independent signalling pathways in the abiotic stress protection in Vitis vinifera
}

\author{
P. VIDIGAL, R. CARVALHO, S. AMÂNCIO, and L. CARVALHO* \\ DRAT/CBAA, Instituto Superior de Agronomia, Universidade Técnica de Lisboa, \\ Tapada da Ajuda, 1349-017 Lisboa, Portugal
}

\begin{abstract}
Peroxiredoxins (Prxs) play major roles in preventing oxidative damage and their function is consistent with the presence of Prx isoforms in most, if not all, cellular compartments and their expression is dependent on environmental conditions. The aim of this study was to identify and characterize genes encoding Prxs in Vitis vinifera. Quantitative real time polymerase chain reaction (qRT PCR) was used to determine their response to irradiance, heat, and water stress. We identified seven vvprx genes, two of which were especially responsive to water stress, followed by heat stress, but no major changes were observed after high irradiance. The vvprxIIF targeted to mitochondria was the most responsive to water stress and it might be involved in drought tolerance through $\mathrm{H}_{2} \mathrm{O}_{2}$ signalling. The vvprxII-2, a putative PrxII, is targeted to the chloroplasts and was the most responsive to heat stress. It might be related with abscisic acid-dependent thermotolerance.
\end{abstract}

Additional key words: abscisic acid, antioxidants, grapevine, heat stress, photosynthesis, qRT PCR, water stress.

\section{Introduction}

Peroxiredoxins (Prxs) are a ubiquitous family of nonheme thiol peroxidases which catalyze the reduction of hydrogen peroxide $\left(\mathrm{H}_{2} \mathrm{O}_{2}\right)$, alkylhydroperoxides, and peroxynitrite to water, alcohols, or nitrite, respectively (Rouhier and Jacquot 2005, Tripathi et al. 2009). These enzymes contain one or two cysteine (cys) residues in their active site and usually function as monomers or dimers. Their common catalytic mechanism involves the catalytic Cys peroxidatic $\left(\mathrm{Cys}_{\mathrm{P}}\right)$ thiol that is oxidized by peroxides to sulfenic acid. The sulfenic acid in the majority of Prxs is reduced by a second Cys resolving $\left(\mathrm{Cys}_{\mathrm{R}}\right)$ thiol forming an intra- or inter-molecular disulfide bond. A new catalytic cycle can only begin after the reduction of the disulfide bond using electron donors such as thioredoxins (Trxs), glutaredoxins (Grxs), or cyclophilins (Dietz et al. 2006). Therefore, Prxs are redox sensitive proteins that can endure reversible oxidationreduction and thus switch 'on' and 'off' depending on the cellular redox state. Thus, they might be involved in balancing signalling cascades mediated by reactive oxygen species (ROS) and in dissipating excessively absorbed energy and protecting the photosynthetic apparatus against oxidative damage (Broin et al. 2002, Dietz et al. 2002, Konig et al. 2002).

In plants, there are four types of Prxs (all nuclear encoded): 1CysPrx, PrxII, 2CysPrx, and PrxQ. Each type plays specific roles according to their spatio-temporal expression patterns and subcellular localizations. Plant Prxs protect the nuclei (1CysPrx), chloroplasts (2CysPrx A, 2CysPrx B, PrxQ, and PrxIIE), cytosol (PrxIIB, PrxIIC, and PrxIID), and mitochondria (PrxIIF) against excess of ROS in stressful conditions, but they are also implicated in redox signalling (Romero-Puertas et al. 2007, Tripathi et al. 2009).

Several genetic approaches have helped to analyze the function of specific Prxs in higher plants or cyanobacteria. Plants deficient in 2CysPrx have shown inhibition of photosynthesis and plant development, decreased chlorophyll accumulation (Baier and Dietz 1999, Baier et al. 2000), and a lower quantum yield of

Received 20 November 2012, accepted 21 March 2013.

Abbreviations: ABA - abscisic acid; APX - ascorbate peroxidase; CAT - catalase; cys - cystein; DHAR - dihydroascorbate reductase; $\mathrm{F}_{\mathrm{v}} / \mathrm{F}_{\mathrm{m}}$ - variable to maximum chlorophyll fluorescence ratio; GOR - glutathione reductase; PPDF - photosynthetic photon flux density; Prx - peroxiredoxin; qRT-PCR - quantitative real time polymerase chain reaction; ROS - reactive oxygen species; SOD - superoxide dismutase, WS - water stress.

Acknowledgements: This research work was supported by project PTDC/AGR-GPL/099624/2008; CBAA (PestOE/AGR/ UI0240/2011) and by the FCT-awarded postdoctoral fellowship SFRH/BPD/43898/2008 to PV.

* Corresponding author; fax: (+351) 213 653383; e-mail: lcarvalho@isa.utl.pt 
photosystem II (PS II) than the wild type plants (Pulido et al. 2010). In the presence of a chloroplast protein synthesis inhibitor, the difference between plants deficient in 2CysPrx and wild types was enhanced which suggested that 2CysPrx can either protect PS II by localized peroxide detoxification or that the interaction between PS II and 2CysPrx affects properties of the photosystem itself, such as stability or turnover (Baier and Dietz 1999).

Plants overexpressing PrxQ are phenotypically similar to wild type plants; nevertheless, they show decreased sensitivity to oxidative stress, small increments in net photosynthetic rate, and increased tolerance to salt and cold stresess (Jing et al. 2006, Lamkemeyer et al. 2006, Petersson et al. 2006). In Arabidopsis thaliana, PrxQ transcripts are the most responsive of the four chloroplast $\operatorname{Prx}$ genes. It was thus suggested that PrxQ has a specific function in protecting photosynthesis different from that of 2CysPrx (Lamkemeyer et al. 2006). Under reducing conditions, $\operatorname{Pr} x Q$ transcripts decrease, and likewise under $\mathrm{H}_{2} \mathrm{O}_{2}$ treatment, they increase (Horling et al. 2002, 2003).

The transcript of 1 CysPrx in the cyanobacterium Synechocystis sp. strain PCC 6803 decreased after $\mathrm{H}_{2} \mathrm{O}_{2}$ treatment and UV-B radiation (Huang et al. 2002, Li et al. 2004, Perez-Perez et al. 2009). In the dehydrationtolerant Xerophyta viscosa, 1CysPrx transcript was not present in the fully hydrated tissue. However, increase of transcript abundance was observed under dehydration, heat, high irradiance, and abscisic acid (ABA) treatment, an indication that 1 CysPrx might play a role in tolerance to extreme drought (Mowla et al. 2002). Using promoter deletions in At-PER1, it was shown that 1 CysPrx was induced by $\mathrm{H}_{2} \mathrm{O}_{2}$, leading to the hypothesis that $1 \mathrm{CysPrx}$ is responsive to oxidative stress (Haslekas et al. 2003).

PrxIIF expression was modified during an interaction between poplar and the rust fungus Melampsora laricii ssp. populina (Gama et al. 2007). However, poplar PrxIIF content did not substantially vary under photo-oxidative conditions or heavy metal treatments (Gama et al. 2007). Also, in Arabidopsis, PrxIIF was unaltered upon changes in irradiance (Horling et al. 2003).

Under oxidative stress, the expression of AtPrxIIE was not changed, however, it rised with increased irradiance and fell when plants were transferred to low irradiance or subjected to ascorbate treatment (Horling et al. 2002, 2003). Expression of AtprxIIC was strongly upregulated by salt, ascorbate, and oxidative treatments (Horling et al. 2002, 2003).

ABA plays a major role in the regulatory network

\section{Materials and methods}

Cuttings from pruned wood of pre-selected grapevines (Vitis vinifera L.) cv. Touriga Nacional were collected from a vineyard in Pegões, $70 \mathrm{~km}$ SE from Lisbon. They were treated with fungicide $(2 \%, \mathrm{~m} / \mathrm{v}$, Benlate $)$ and kept at $4{ }^{\circ} \mathrm{C}$ for 2 months and then treated again with fungicide before rooting. Rooting took place in distilled controlling At2cysPrx expression (Baier et al. 2004). Since ABA is involved in stress adaptation (Song et al. 2012), interestingly it functions as a suppressor of 2 cysPrx expression as revealed from work with ABAinsensitive and ABA-deficient mutants combined with ABA feeding (Baier et al. 2004). This observation links 2CysPrx to a function in regular photosynthesis rather than in stress response (Dietz 2007). Promoter analyses for cis-regulatory elements identified the ABAresponsive element ABRE in Arabidopsis 1CysPrxs (AtPER1). In plants ectopically expressing ABI3, AtPER $1: \because G U S$ expression was found in leaves and AtPER 1 was induced by ABA and $\mathrm{H}_{2} \mathrm{O}_{2}$ (Haslekas et al. 1998).

When considering the constituents of the antioxidative machinery, Prxs are not considered to be enzymes in the first line of defence. A prominent part of the extensive antioxidant network consists of enzymes such as superoxide dismutase (SOD) scavenging superoxide radical and ascorbate peroxidase (APX) and catalase (CAT) scavenging $\mathrm{H}_{2} \mathrm{O}_{2}$ (Asada 1999). These enzymes work in tandem or complementary, as it is the case of APX and CAT, the former with high affinity to $\mathrm{H}_{2} \mathrm{O}_{2}$, performing a crucial role in the control of ROS in each compartment, whereas the latter with its low affinity to $\mathrm{H}_{2} \mathrm{O}_{2}$ and presence mainly in peroxisomes is associated with processing $\mathrm{H}_{2} \mathrm{O}_{2}$ generated in photorespiration and in situations of excessive $\mathrm{H}_{2} \mathrm{O}_{2}$ formation (Mittler 2002, Vandenabeele et al. 2004). Prxs are described as interacting with these enzymes scavenging $\mathrm{H}_{2} \mathrm{O}_{2}$ and lipid peroxides and tuning their content in signalling events (Dietz 2011).

This study was designed to comprehensively analyse, for the first time in $V$. vinifera, the Prx gene family. For that purpose, we identified seven Prx genes and determined their expression under abiotic stresses. A relationship between their expression, the behaviour of other elements of the antioxidative network, and physiological responses after each abiotic stress was established. Also, the involvement of different Prxs in different stress signalling pathways was established: vvprxIIF in drought tolerance through $\mathrm{H}_{2} \mathrm{O}_{2}$ signalling and vvprxII-2 in ABA involved thermotolerance. The study of the response of these genes under abiotic stress is paramount because different Vitis species differ in their response to stress (Upadhyay et al. 2012) and in a Mediterranean environment with high irradiance, high temperature, and low water supply during the summer growing season, oxidative stress is prone to occur.

water supplemented with nutrient solution (Rhue et al. 1978) in the dark, until full rooting and shoot elongation. The saplings were transferred to pots filled with disinfected soil and maintained in the greenhouse under irradiance of $200 \mu \mathrm{mol} \mathrm{m} \mathrm{m}^{-2} \mathrm{~s}^{-1}$, a $16-\mathrm{h}$ photoperiod, day/night temperature of $25 \pm 2 / 23 \pm 2{ }^{\circ} \mathrm{C}$ and relative 
humidity of $c a .60 \%$. Plants were watered with nutrient solution whenever necessary.

When plants were $c a$. 4-month-old and $70 \mathrm{~cm}$ high, they were exposed to different stresses: 1) high photosynthetic photon flux density (PPFD) of $2000 \mu \mathrm{mol} \mathrm{m}{ }^{-2} \mathrm{~s}^{-1}$ for $1 \mathrm{~h}$ (high irradiance, $\mathrm{HI}$ ); 2) temperature of $42 \pm 1{ }^{\circ} \mathrm{C}$ for $1 \mathrm{~h}$ (heat stress, HS); and 3) to water stress (WS) induced by cessation of watering till pre-dawn leaf water potential reached $-0.9 \mathrm{MPa}$ (pressure chamber, Model 600, PMS Instruments Company, Albany, OR, USA). The third, fourth, and fifth leaves were harvested immediately after the measurements of photosynthetic and chlorophyll fluorescence parameters and stored at $-80^{\circ} \mathrm{C}$ until use.

$\mathrm{H}_{2} \mathrm{O}_{2}$ production was assayed following the spectrophotometric method described by Carvalho et al. (2006) using the peroxide-mediated oxidation of $\mathrm{Fe}^{2+}$ followed by the reaction of $\mathrm{Fe}^{3+}$ with xylenol orange. This method produces reproducible results in the $0.1-1 \mathrm{mM} \mathrm{H}_{2} \mathrm{O}_{2}$ concentration range.

The extracts for the measurement of ABA were carried out as described by Vilela et al. (2007). ABA was quantified through immunoassay by indirect enzymelinked immunosorbent assay (ELISA) with monoclonal antibodies using a commercial kit (Olchemim Enzyme Immunoassay, Olomouc, Czech Republic) according to the manufacturer's recommendations.

The extraction for measuring Prx activity was performed at $4{ }^{\circ} \mathrm{C}$ from $0.5 \mathrm{~g}$ of frozen leaf material according to Carvalho et al. (2006). The material was ground using a mortar and pestle with $50 \%(\mathrm{~m} / \mathrm{v})$ polyvinylpolypyrrolidone. The extraction buffer was $0.2 \mathrm{M}$ potassium phosphate, $\mathrm{pH} 8.0$, containing $1 \mathrm{mM}$ ethylenediaminetetraacetic acid $\left(\mathrm{Na}_{2}\right.$-EDTA), $1 \mathrm{mM}$ dithiothreitol (DTT), $10 \mathrm{mM} \mathrm{MgCl}_{2}$, and $0.2 \mathrm{mM}$ phenylmethanesulfonylfluoride (PMSF). Extracts for all quantifications were centrifuged at $27000 \mathrm{~g}$ and $4{ }^{\circ} \mathrm{C}$ for $10 \mathrm{~min}$ and the supernatants were desalted through $P D-10$ columns ( $G E$ Healthcare LifeSciences, Buckinghamshire, UK). Protein was quantified by the method of Bradford
(1976) using a commercial kit (Bio-Rad, Hercules, CA).

Reduction of $\mathrm{H}_{2} \mathrm{O}_{2}$ by $V$. vinifera Prxs was quantified in vitro using a non-enzymatic, DTT-dependent activity assay by measuring the decrease in $\mathrm{H}_{2} \mathrm{O}_{2}$ concentration in the assay solution as previously described (Horling et al. 2003, Aragón et al. 2009). The assay contained $100 \mathrm{mM}$ K-Pi buffer ( $\mathrm{pH} 7.0$ ), 0.3 to $3 \mu \mathrm{M}$ Prx, $10 \mathrm{mM}$ DTT, and $100 \mu \mathrm{M} \mathrm{H}_{2} \mathrm{O}_{2}$ in a total volume of $1 \mathrm{~cm}^{3}$. The reaction was initiated with $\mathrm{H}_{2} \mathrm{O}_{2}$ and stopped with $0.8 \mathrm{~cm}^{3}$ of $12.5 \%(\mathrm{~m} / \mathrm{v})$ trichloroacetic acid to an aliquot of $0.05 \mathrm{~cm}^{3}$ of the assay solution. After adding $0.2 \mathrm{~cm}^{3}$ of $10 \mathrm{mM} \mathrm{Fe}\left(\mathrm{NH}_{4}\right)_{2}\left(\mathrm{SO}_{4}\right)_{2}$ and $0.1 \mathrm{~cm}^{3}$ of $2.5 \mathrm{M} \mathrm{KSCN}$, the absorbance at $480 \mathrm{~nm}$ was measured to quantify the $\mathrm{H}_{2} \mathrm{O}_{2}$ content of the solution, and $\mathrm{H}_{2} \mathrm{O}_{2}$ reduction rate was calculated.

Net photosynthetic rate $\left(\mathrm{P}_{\mathrm{N}}\right)$ was measured using a Li-6400 portable photosynthesis system fitted with a leaf chamber mounted with a light source (LiCor, Lincoln, Nebraska, USA). The temperature inside the leaf cuvette was set to $25 \pm 2{ }^{\circ} \mathrm{C}$. The response of $\mathrm{P}_{\mathrm{N}}$ to irradiance (from 0 to $2000 \mu \mathrm{mol} \mathrm{m} \mathrm{m}^{-2} \mathrm{~s}^{-1}$ ) was performed at ambient $\mathrm{CO}_{2}$ concentration ( $c$ a. $350 \mu \mathrm{mol} \mathrm{mol}{ }^{-1}$ ). Chlorophyll fluorescence parameters were quantified with a portable modulated fluorometer (Mini-Pam, Walz, Effeltrich, Germany). The parameters measured were the minimum fluorescence of dark-adapted leaves $\left(\mathrm{F}_{0}\right)$, maximum fluorescence of dark-adapted leaves $\left(\mathrm{F}_{\mathrm{m}}\right)$ after a saturation pulse with an irradiance of $1800 \mu \mathrm{mol} \mathrm{m}^{-2} \mathrm{~s}^{-1}$ to calculate the variable fluorescence $\left(\mathrm{F}_{\mathrm{v}}=\mathrm{F}_{\mathrm{m}}-\mathrm{F}_{0}\right)$ and the maximum quantum efficiency of PS II photochemistry in dark-adapted leaves $\left(\mathrm{F}_{\mathrm{v}} / \mathrm{F}_{\mathrm{m}}\right)$.

Total RNA was extracted according to the modified method of Geuna et al. (1998), treated with RQ1 RNasefree DNase (Promega, Madison, WI, USA), and reversetranscribed using oligoDT 20 and Superscript II RNase H-reverse transcriptase (Invitrogen, Carlsbad, CA, USA) according to the manufacturer's recommendations. RT qPCR was performed as previously described Coito et al. (2012) and the primers used are listed in Table 1.

Table 1. Primers used for the qRT PCR of the $V$. vinifera peroxiredoxins and of the ascorbate-glutathione cycle genes.

\begin{tabular}{|c|c|c|c|}
\hline Primer & Accession No. & $\begin{array}{l}\text { Sequence }\left(5^{\prime}-3^{\prime}\right) \\
\text { forward }\end{array}$ & reverse \\
\hline$v v 2 c y s p r x 01$ & JN392719 & TCCTCTCGTCTAACCATTCCTCATC & СТCAAACTCTTCATAGCGGTCACTG \\
\hline vvprxQ & JN392725 & АССТTССТСАСТСТTAATGGCTTCC & CTTTCCTCACCTTGTTTCCGTCATC \\
\hline vvprxII-1 & JN392721 & ATCTTCCGATTTCACATCCACCATC & TCACCTGTTCCTTCGTCTTTCCTTG \\
\hline vvprxII-2 & JN392722 & TTTCTCTCTCCACTACGATCTTGCC & CTCCTCTTGTCACGGTATCCCTTTG \\
\hline vvprxIIE & JN392723 & AATCTACCATAGGAATGCTCGTTGC & AATCAGACACAGGAAACCACAAACC \\
\hline vvprxIIF & JN392724 & CGAAGCATGATGATGAAATCAACGG & GCACCAGAAACCTTAACCTCGGATG \\
\hline vv1cysprx03 & JN392718 & GAAACCACACATGGGAAGTTCAAAC & GGTATGACTTGTTGGATGGAAGAGC \\
\hline$D H A R$ & XM_002282363.1 & TAATGACGGATCCGAGAAGG & CTGGTCAAGCTCTCAGGGAC \\
\hline GOR2 & XM_002285636.1 & GTGCAGTCAAACTGTGCCTTAG & GAGCGAAGAACAGGCTACAGAT \\
\hline CuZnSOD & XM_002274385.1 & GTCATGCGGGTGACCT & AGATTGGCATGTGGTGT \\
\hline$M n S O D$ & $\mathrm{AB} \bar{X} 79342.1$ & CCTTACGATTATGGCGCATT & CTTCACTGGAAGGAGCAAGG \\
\hline$C A T$ & AF236127 & ATGGATCCTTACAAGTATCGTC & GAGGTCACTCACGATGTCTC \\
\hline$A P X 3$ & XM 002278245.1 & ATGGCTGCACCGATTGTTGATGCG & GAACGAAGAAGAGCACACTCATG \\
\hline Act2 & AF369525.1 & TGGATTCTGGTGATGGTGTGAGTC & CAATTTCCCGTTCAGCAGTAGTGG \\
\hline
\end{tabular}


To compare data from different PCR runs or cDNA samples, $\mathrm{C}_{\mathrm{T}}$ values were normalized to the $\mathrm{C}_{\mathrm{T}}$ value of Act2, a gene that was found to be a good reference in similar experimental conditions (Coito et al. 2012).

BLASTP and TBLASTX searches were performed using other sequenced plant species available (Arabidopsis thaliana, Oryza sativa, and Populus trichocarpa) against $V$. vinifera in GenBank (http:// www.ncbi.nlm.nih.gov/blast/Blast.cgi; Benson et al. 2011) and Genoscope (www.genoscope.cns.fr). The sequences of $V$.vinifera thus obtained were screened for the conserved thioredoxin fold using the conserved domain database $C D D$ (www.ncbi.nlm.nih.gov/ Structure/cdd/cdd.shtml; Marchler-Bauer et al. 2011). Each vvprx identified was cloned with pMOSBlue Blunt ended PCR cloning kit (GE Healthcare, Life Sciences) and the sequenced fragments (STAB-Vida, Oeiras, Portugal) compared to the expected sequences using CLUSTALW2.0 (Larkin et al. 2007, http://www.ebi.ac.uk/ Tools/msa/clustalw2/). The complete sequences were then submitted to GenBank and their accessions are listed in Table 2. All the other genes studied were already available in $N C B I$ database.

The phylogenetic tree was calculated based on ClustalW2.0 alignments and using the neighbor-joining method (Saitou and Nei 1987). The quality of the predicted tree was tested by calculating the bootstrap values based on 1000 replicates (Felsenstein 1985). Branches corresponding to partitions reproduced in less than $50 \%$ bootstrap replicates were excluded. The distances were computed using the Dayhoff matrix based method (Schwarz and Dayhoff 1979). The analyses were conducted in MEGA5 (Tamura et al. 2007).

To predict the subcellular localization sites of VvPrxs, their amino acid sequences were analysed first by TargetP (www.cbs.dtu.dk/services/TargetP/) followed by analysis in WoLFPSORT (http://wolfpsort.org/, Horton et al. 2007) in order to verify the results. Theoretical determination of each VvPrx molecular mass $(\mathrm{kDa})$ and isoelectric point was computed in http://web.expasy.org/ compute pi/ (Gasteiger et al. 2005).

The following parameters were subjected to statistical analysis by one-way ANOVA using GraphPad Prism v. 5.04 for Windows (GraphPad Software, La Jolla, CA, USA). Five replicates for photosynthesis and chlorophyll fluorescence, three replicates for gene expression and enzyme activity, and six replicates for ABA and $\mathrm{H}_{2} \mathrm{O}_{2}$ were used. In order to analyze the relationship between Prx activity and $\operatorname{Prx}$ gene expression with $\mathrm{H}_{2} \mathrm{O}_{2}$ content, ABA content, and $\mathrm{F}_{\mathrm{v}} / \mathrm{F}_{\mathrm{m}}$ under each abiotic stress, $\mathrm{R}^{2}$ from the Pearson correlation analysis was calculated and statistically analysed with GraphPad Prism v. 5.04.

Table 2. The seven $V$. vinifera Prx genes cloned and annotated, with their predicted protein properties.

\begin{tabular}{|c|c|c|c|c|c|}
\hline Gene & Accession & Type & Number of amino acids & $\mathrm{M}_{\mathrm{r}}[\mathrm{kDa}]$ & $\mathrm{pI}$ \\
\hline vvlcysprx03 & JN392718 & 1 cys peroxiredoxin & 183 & 20.22 & 7.85 \\
\hline$v v 2$ cysprx 01 & JN392719 & 2 cys peroxiredoxin & 274 & 30.27 & 6.84 \\
\hline vvprxII-1 & JN392721 & type II peroxiredoxin & 256 & 28.11 & 6.82 \\
\hline vvprxII-2 & JN392722 & type II peroxiredoxin & 254 & 27.56 & 5.15 \\
\hline vvprxIIE & JN392723 & type II peroxiredoxin & 212 & 22.51 & 7.67 \\
\hline vvprxIIF & JN392724 & type II peroxiredoxin & 201 & 21.83 & 8.74 \\
\hline vvprxQ & JN392725 & peroxiredoxin $\mathrm{Q}$ & 214 & 23.47 & 9.68 \\
\hline
\end{tabular}

\section{Results}

The $V$. vinifera Prx family includes at least seven genes (Table 2). The inclusion of these genes in their respective Prx type was confirmed by phylogentic analyses (Fig. 1). According to the number and position of conserved Cys residues, the VvPrx were classified as follows: VvPrxIIE, VvPrxIIF, VvPrxII-1, and VvPrxII-2 are type II Prx proteins, $\mathrm{Vv} 1 \mathrm{Cys} P \mathrm{x} 03$ is a $1 \mathrm{CysPrx}$ protein, Vv2CysPrx01 is a 2CysPrx protein, and VvPrxQ belongs to PrxQ (Fig. 1). The open reading frames (ORFs) of these VvPrxs encoded polypeptides of 162 - 274 amino acids with predicted molecular masses ranging from 20.22 to $30.27 \mathrm{kDa}$ and $\mathrm{pI}$ of 5.15 to 9.68 (Table 2). Furthermore, in silico analyses predicted that Vv2CysPrx01, VvPrxQ, VvPrxIIE, VvPrxII-1, and VvPrxII-2 are targeted to the chloroplasts, Vv1CysPrx03 to the cytosol, and VvPrxIIF to the mitochondria.
Prxs play major roles in preventing oxidative damage and their function is consistent with the presence of Prx isoforms in most, if not all, cellular compartments. Transcription of Prx genes depends on environmental factors (Dietz et al. 2006). In $V$. vinifera, the genes encoding choroplastic (vv2cysprx01 and vvprxII-1) and mitochondrial (vvprxIIF) Prx isoforms showed the highest expression after HS and WS.

After HI, all vvprxs showed low induction comparatively to the ascorbate-glutathione cycle genes. The highest transcription after HI was measured in the peroxisome, with a 12 -fold induction of $A P X 3$, and in the mitochondria with $c a$. 2-fold induction of $M n S O D$ (Table 3). In the peroxisome, CAT expression was unchanged. However, in the chloroplast, $C u Z n S O D$ was slightly repressed as well as the cytosol GOR2. 


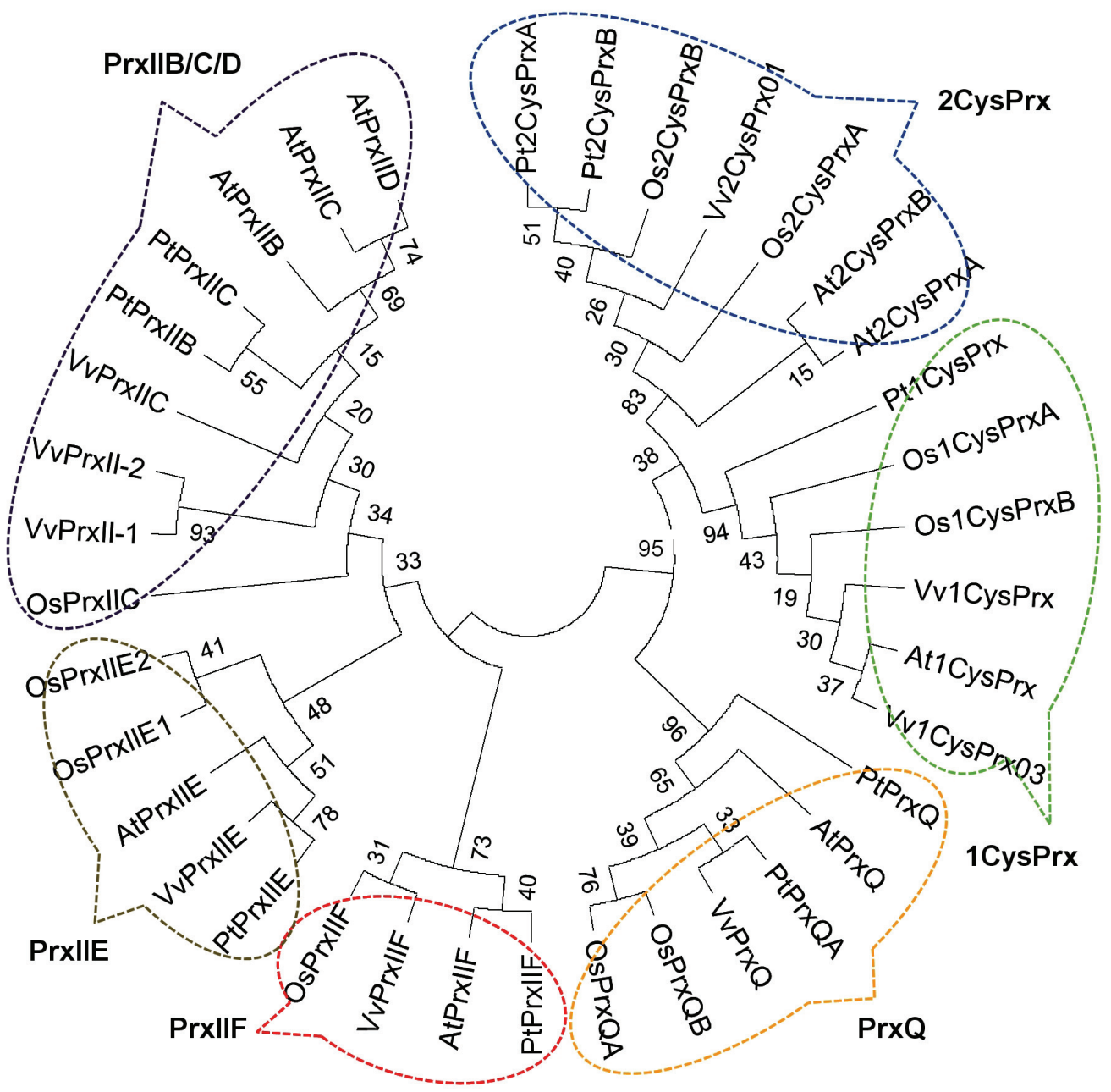

Fig. 1. Comparative analyses of Prx genes between Arabidopsis thaliana (At), Populus trichocarpa (Pt), Oryza sativa (Os), and Vitis vinifera $(V v)$, conducted in $M E G A 5$.

The gene coding the chloroplast isoform VvPrxII-2 showed $c a$. 2-fold induction after HS (Table 4). Of the six genes involved in the ascorbate-glutathione cycle, five were up-regulated after HS (Table 3). The highest

Table 3. Relative expression of ascorbate-glutathione cycle genes after high irradiance (HI), heat stress (HS), and water stress (WS). Data are $\log _{2}$ expression ratios of stress treatments in relation to control conditions. Means \pm SE of three independent biological replicates. * - indicate significant differences between control and treatment conditions at $P<0.05$ (ANOVA).

\begin{tabular}{lccc}
\hline Gene name & \multicolumn{2}{l}{ HI } & \multicolumn{2}{l}{ HS } & WS \\
\hline CAT & $0.8 \pm 0.00$ & $4.6 \pm 0.00^{*}$ & $-0.2 \pm 0.00$ \\
APX3 & $12.4 \pm 0.00$ & $14.0 \pm 0.00^{*}$ & $0.4 \pm 0.00$ \\
GOR2 & $-0.6 \pm 0.02$ & $-0.7 \pm 0.01^{*}$ & $0.0 \pm 0.00$ \\
DHAR & $-0.3 \pm 0.00$ & $3.5 \pm 0.00$ & $5.7 \pm 0.00^{*}$ \\
MnSOD & $1.7 \pm 0.00$ & $5.7 \pm 0.00^{*}$ & $6.5 \pm 0.00^{*}$ \\
CuZnSOD & $-0.8 \pm 0.01^{*}$ & $1.9 \pm 0.00^{*}$ & $-1.2 \pm 0.01^{*}$ \\
\hline
\end{tabular}

up-regulation was the 14-fold induction of $A P X 3$ observed in the peroxisomes, followed by $M n S O D$ with $c a$. 6-fold induction in the mitochondria, and $C A T$ with $c a$. 5-fold induction in the peroxisomes. In the cytosol, $D H A R$ was $c a$. 4-fold up-regulated, but GOR2 was slightly repressed. In the chloroplast, $C u Z n S O D$ was upregulated by $c$. 2 -fold.

The gene coding for the mitochondrial Prx isoform showed the highest up-regulation after WS (Table 4) with 3 -fold induction, followed by vv2cysprx01, vvprxIIE, and vvprxII-1 in the chloroplast, and vvlcysprx 03 in the cytosol with $\mathrm{ca}$. 2-fold induction. The transcriptions of vvprxII-2 and vvprxQ were unchanged. The highest transcript levels were measured in $M n S O D$ (6.5-fold) in the mitochondria and in DHAR in the cytosol (5.7-fold). Neither the cytosol GOR2 nor peroxisome CAT changed. In the chloroplast, CuZnSOD was slightly downregulated.

In the activity assay with a DTT-based non-enzymatic regeneration system, Prxs showed $\mathrm{H}_{2} \mathrm{O}_{2}$ scavenging activity decreasing after stress. Under control conditions, 
Table 4. Relative expression of vvprxs after high irradiance (HI), heat strass (HS), and water stress (WS). For each gene, the upper-line corresponds to $\log _{2}$ expression ratios of stress treatments in relation to control conditions (means \pm SE of three independent biological replicates) and the lower line shows correlation coefficient $R^{2}$ between gene expression and Prx activity, ABA content, and $\mathrm{H}_{2} \mathrm{O}_{2}$ content (Prx $|\mathrm{ABA}| \mathrm{H}_{2} \mathrm{O}_{2}$ ). * - indicate significant differences between control and treatment at $P<0.05$; \# - indicate significant differences in the Pearson correlation at $P<0.05$.

\begin{tabular}{llll}
\hline Gene name & HI & HS & WS \\
\hline vvlcysprx03 & $-1.0 \pm 0.13^{*}$ & $1.3 \pm 0.05^{*}$ & $1.8 \pm 0.15^{*}$ \\
& $0.7|0.7| 0.3$ & $0.1|0.1| 0.2$ & $0.5\left|0.8^{\#}\right| 0.5$ \\
vv2cysprx01 & $-1.0 \pm 0.05$ & $0.8 \pm 0.01^{*}$ & $2.2 \pm 0.05^{*}$ \\
& $0.2|0.2| 0.8^{\#}$ & $0.1|0.1| 0.3$ & $0.3|0.7| 0.6$ \\
vvprxII-1 & $-0.2 \pm 0.21$ & $1.0 \pm 1.03$ & $1.8 \pm 1.82$ \\
& $0.8^{\#}\left|0.8^{\#}\right| 0.2$ & $0.0|0.2| 0.1$ & $0.3|0.7| 0.4$ \\
vvprxII-2 & $-2.2 \pm 1.15$ & $1.8 \pm 0.21^{*}$ & $0.9 \pm 0.38^{*}$ \\
& $1.0^{\#}|0.0| 1.0^{\#}$ & $1.0^{\#}\left|1.0^{\#}\right| 0.6$ & $0.5|0.1| 0.8^{\#}$ \\
vvprxIIE & $-1.3 \pm 0.11$ & $0.4 \pm 0.04$ & $1.9 \pm 0.09$ \\
& $0.5|0.4| 0.6$ & $0.2|0.1| 0.3$ & $0.1|0.5| 0.6$ \\
vvprxIIF & $-0.9 \pm 0.07^{*}$ & $1.4 \pm 0.01^{*}$ & $2.9 \pm 0.05^{*}$ \\
& $0.1|0.1| 0.9^{\#}$ & $0.4|0.0| 0.6$ & $0.6\left|0.9^{\#}\right| 0.9^{\#}$ \\
vvprxQ & $-1.8 \pm 0.14$ & $-1.1 \pm 0.09^{*}$ & $0.5 \pm 0.04^{*}$ \\
& $0.0|0.0| 1.0^{\#}$ & $0.1|0.1| 0.2$ & $0.7|0.3| 0.5$ \\
\hline
\end{tabular}

the Prx activity was $56.4 \mathrm{nmol}\left(\mathrm{H}_{2} \mathrm{O}_{2}\right) \mu \mathrm{g}^{-1}$ (prot.) $\min ^{-1}$ (Table 5). The greatest decrease was after HI $(84 \%$ decrease of total Prx activity). After HS, there was a $49 \%$ decrease. The smallest decrease was after WS corresponding to only $3 \%$ decrease (Table 5 ).

After $\mathrm{HI}$, there was a decrease in both $\mathrm{H}_{2} \mathrm{O}_{2}$ and ABA content of 18 and $55 \%$, respectively (Table 5). However, after HS, there was a $59 \%$ increase of ABA content but a $29 \%$ decrease of $\mathrm{H}_{2} \mathrm{O}_{2}$ content. The highest increase of $\mathrm{H}_{2} \mathrm{O}_{2}$ content was measured after WS (51\% increase), while ABA content remained unchanged.

Plants showed higher $\mathrm{P}_{\mathrm{N}}$ after HI and HS (Fig. 2) but no significant differences were observed in PS II efficiency in dark adapted leaves $\left(\mathrm{F}_{\mathrm{v}} / \mathrm{F}_{\mathrm{m}}\right.$; Table 5). Actually, there were no major changes in PS II efficiency in response to the various stresses.

\section{Discussion}

The Prx family is ubiquitous in all organisms from bacteria to higher plants. It is a small gene family with 10 genes in Arabidopsis and rice and 9 in poplar. In this study, seven $V$. vinifera $\operatorname{Prx}$ genes were cloned, one $v v 1$ cysprx, one $v v 2$ cysprx, one vvprxQ, and four vvprxII that include two putative new vvprxs. Most of the genes coding for Prx isoforms in $V$. vinifera were significantly up-regulated after HS and WS. As opposite to this response, the vvprx genes were found to be downregulated after $\mathrm{HI}$ as previously observed Carvalho et al. (2011).
Table 5. Total Prx activity [nmol $\left(\mathrm{H}_{2} \mathrm{O}_{2}\right) \mu \mathrm{g}^{-1}$ (prot.) $\left.\min ^{-1}\right], \mathrm{H}_{2} \mathrm{O}_{2}$ content $\left[\mu \mathrm{mol} \mathrm{g}{ }^{-1}\right.$ (f.m.)], ABA content $\left[\mathrm{nmol} \mathrm{g}^{-1}\right.$ (d.m.)], and maximum quantum efficiency of PS II photochemistry $\left(\mathrm{F}_{\mathrm{v}} / \mathrm{F}_{\mathrm{m}}\right)$ in $V$. vinifera under control conditions and after high irradiance (HI), heat stress (HS), and water stress (WS). Means \pm SE of 3 - 6 independent biological replicates; * indicate significant differences between stress treatments relative to control at $P<0.05$. A Pearson correlation analysis was made in order to analyze the relationship between Prx activity and the other data in the table, however, no significant correlations were found.

\begin{tabular}{lrrrr}
\hline & \multicolumn{1}{c}{$\mathrm{C}$} & \multicolumn{1}{c}{ HI } & \multicolumn{1}{l}{ HS } & WS \\
\hline Prx & $56.4 \pm 0.00$ & $9.2 \pm 0.00 *$ & $28.5 \pm 0.00 *$ & $54.7 \pm 0.00$ \\
$\mathrm{H}_{2} \mathrm{O}_{2}$ & $12.9 \pm 1.50$ & $10.6 \pm 1.20 *$ & $9.1 \pm 0.80 *$ & $19.5 \pm 2.80 *$ \\
$\mathrm{ABA}$ & $2.9 \pm 0.80$ & $1.3 \pm 0.80 *$ & $4.6 \pm 0.40 *$ & $2.5 \pm 0.40$ \\
$\mathrm{~F}_{\mathrm{v}} / \mathrm{F}_{\mathrm{m}}$ & $0.8 \pm 0.01$ & $0.8 \pm 0.01$ & $0.7 \pm 0.02$ & $0.8 \pm 0.03$ \\
\hline
\end{tabular}

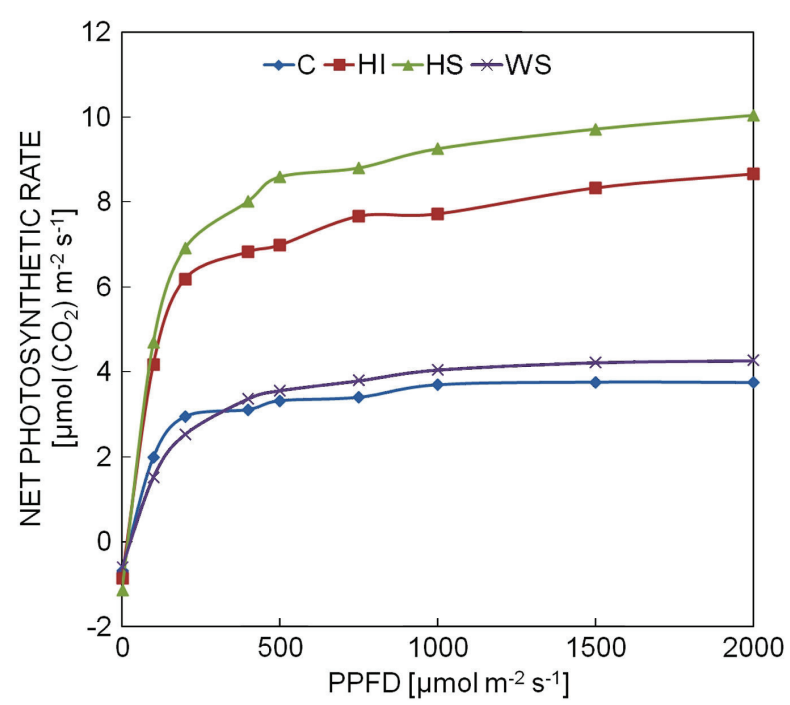

Fig. 2. Response of $\mathrm{P}_{\mathrm{N}}$ to irradiance under control conditions (C) and after high irradiance (HI), heat stress (HS), and water stress (WS). Means of five independent biological replicates. PPFD - photosynthetic photon flux density.

As a preliminary study to understand the function of these VvPrxs in grapevine under abiotic stresses, we used bioinformatic tools to predict their subcellular localization based on the N-terminal signal sequence of each protein: vv2cysprx01, vvprxQ, vvprxIIE, vvprxII-1, and vvprxII-2 were targeted to the chloroplasts and vvprxIIF to the mitochondria. The vvlcysprx 03 was targeted to the nucleus and the cytosol consistently with Arabidopsis 1CysPrx (Haslekas et al. 2003, Pulido et al. 2009).

Among the seven vvprx genes, vvprx $Q$ was repressed or did not change its transcription after the abiotic stresses 
applied. However, previous studies in Arabidopsis showed upregulation of AtPrxQ after oxidative stress (Horling et al. 2003). PrxQ is considered the most responsive chloroplast Prx-transcript in Arabidopsis (Dietz 2011). In $V$. vinifera after $\mathrm{HI}$, the transcription of the chloroplast genes was in general down-regulated indicating the absence of oxidative stress in the chloroplast and, therefore, explaining the repression of $v$ vprx $Q$ which showed a strong positive correlation with $\mathrm{H}_{2} \mathrm{O}_{2}$ (Table 4). The vvprxII-2 was the gene that showed the lowest transcription which was proven by a positive correlation with Prx activity under HI.

After HS, CuZnSOD was 2-fold induced (Table 3) indicating a mild oxidative stress in the chloroplasts but vvprxQ remained repressed (Table 4), most likely due to the induction of the other chloroplast vvprx such as vvprxII-2 which might replace vvprxQ under these conditions. The vvprxII-2 had a strong positive correlation with Prx activity and ABA under HS (Table 4) signifying a strong influence of this gene on the activity of Prx under stress and that it also might be involved in the ABA signalling pathway. Interestingly, there was a significant increase of $\mathrm{ABA}$ under $\mathrm{HS}$ which coincided with the decrease of $\mathrm{H}_{2} \mathrm{O}_{2}$ (Table 5).

The photosynthesis of the plants after the three stresses applied in this study showed a tendency to higher rates than under control conditions (Fig. 2) although this probably reflects a transient response since these mostly short term stresses did not affect PS II (Table 5, $\mathrm{F}_{\mathrm{v}} / \mathrm{F}_{\mathrm{m}}$ values). After $\mathrm{HS}$, there was a significant increase in transcription of peroxisome genes, $C A T$ and $A P X 3$, and of $M n S O D$ in the mitochondria (Table 3). In addition, there was an $18 \%$ decrease in $\mathrm{H}_{2} \mathrm{O}_{2}$ content which goes in concordance with the increase in transcripts of the genes coding for $\mathrm{H}_{2} \mathrm{O}_{2}$ scavenging enzymes such as CAT and APX 3 and of the gene coding for MnSOD which leads to the generation of $\mathrm{H}_{2} \mathrm{O}_{2}$. There was a significant increase in ABA content after HS (Table 5) as Wang et al. (2005) also reported in young $V$. vinifera plants subjected to heat. These results, together with the photosynthetic response could indicate that ABA can be involved in $V$. vinifera response to $\mathrm{HS}$ as well as in improved thermotolerance. An in silico analysis revealed that the promoter of vvprxII-2 contains the ABAresponsive element ABRE. As already mentioned, vvprxII-2 was the most induced vvprx gene under HS, thus it is possible to admit that VvPrxII-2 might be involved in ABA signalling in $V$. vinifera thermotolerance.

From all the abiotic stresses applied, WS caused the most intense changes in the transcription of vvprx genes. Even though there was no negative effect on the photosynthetic capacity, there was a significant increase in $\mathrm{H}_{2} \mathrm{O}_{2}$ content (Table 5) accompanied by the 6-fold upregulation of $M n S O D$ (Table 3). The vvprxIIF sustained significantly up-regulated but no changes were observed in the peroxisome $C A T$ and $A P X 3$. Sweetlove et al.
(2002) showed that PrxIIF detoxifies $\mathrm{H}_{2} \mathrm{O}_{2}$ released from superoxide dismutation by MnSOD. This, together with the high transcription of $C A T$ in PrxIIF knock-out plants, suggests that vvprxIIF could play a role in the tolerance of $V$. vinifera to drought, that is also further confirmed by the strong correlation between vvprxIIF expression and ABA and $\mathrm{H}_{2} \mathrm{O}_{2}$ content (Table 4). In the cytosol, vvlcysprx03 was up-regulated as well as vvprxII-1, vvprxIIE, and $v v 2$ cysprx 01 in the chloroplasts indicating their roles in tolerance to drought. Similar results were observed in the dehydration-tolerant Xerophyta viscosa where 1 CysPrx increased under dehydration indicating that 1 CysPrx also plays a role in tolerance to extreme drought (Mowla et al. 2002).

Prx enzymes have a low catalytic efficiency towards $\mathrm{H}_{2} \mathrm{O}_{2}$ and thus it is believed that they are only effective to eliminate low concentrations of $\mathrm{H}_{2} \mathrm{O}_{2}$ (Rhee et al. 2005). However, it was shown that the catalytic efficiency of the bacterial Prx $(\mathrm{AhpC})$ can be as high as in other enzymes such as CAT (Parsonage et al. 2008). However, CAT is mainly located in peroxisomes and Prxs are present in multiple subcellular localizations. It is suggested that their ubiquitous distribution meets the requirements for local $\mathrm{H}_{2} \mathrm{O}_{2}$ detoxification in general antioxidant defence and also allows their functions as redox sensors and redox elements in signalling networks (Dietz 2011). Preliminary results using virus induced gene silencing (VIGS) in Nicotiana benthamiana showed that the silencing of Prx genes from different subcellular locations has different responses, such as a higher $\mathrm{H}_{2} \mathrm{O}_{2}$ accumulation when the chloroplast gene is silenced in comparison with the cytosol gene (data not shown).

In the current study in $V$. vinifera after WS, $\mathrm{H}_{2} \mathrm{O}_{2}$ increased but there was a decrease in Prx activity that could be due to the inactivation of the peroxidase activity of Prxs and the triggering of signalling events that led to the stress responses, such as the up-regulation of DHAR (Table 3). In fact, several studies have established a regulatory role of DHAR during oxidative stress tolerance and acclimation (Hossain and Fujita 2011). Actually, Wood et al. (2003) suggested that when $\mathrm{H}_{2} \mathrm{O}_{2}$ accumulates to large levels, inactivation of Prx through hyperoxidation occurs, thereby facilitating redoxdependent signalling, a concept known as the "floodgate" hypothesis.

In this work, we identified seven $V$. vinifera Prx genes and determined their expression under various abiotic stresses. WS produced the most extreme responses followed by HS and no changes were observed after HI. The vvprxIIF and vvprxII-2 were the most responsive Prxs after WS and HS, respectively. It is proposed that VvPrxII-2 can be involved in thermotolerance through ABA signalling and that VvPrxIIF might play an important role in drought tolerance in V.vinifera, although more studies must be undertaken to confirm these hypotheses. 


\section{References}

Aragón, C., Carvalho, L.C., González, J., Escalona, M., Amâncio, S.: Sugarcane (Saccharum sp. hybrid) propagated in headspace renovating systems shows autotrophic characteristics and develops improved anti-oxidative response. - Trop. Plant Biol. 2: 38-50, 2008.

Asada, K.: The water-water cycle in chloroplasts: scavenging of active oxygens and dissipation of excess photons. - Annu. Rev. Plant Physiol. Plant mol. Biol. 50: 601-639, 1999.

Baier, M., Dietz, K.J.: Protective function of chloroplast 2-cysteine peroxiredoxin in photosynthesis. Evidence from transgenic Arabidopsis. - Plant Physiol. 119: 1407-1414, 1999.

Baier, M., Noctor, G., Foyer, C.H., Dietz, K.J.: Antisense suppression of 2-cysteine peroxiredoxin in Arabidopsis specifically enhances the activities and expression of enzymes associated with ascorbate metabolism but not glutathione metabolism. - Plant Physiol. 124: 823-832, 2000.

Baier, M., Stroher, E., Dietz, K.J.: The acceptor availability at photosystem I and ABA control nuclear expression of 2-Cys peroxiredoxin-A in Arabidopsis thaliana. - Plant Cell Physiol. 45: 997-1006, 2004.

Benson, D.A., Karsch-Mizrachi, I., Lipman, D.J., Ostell, J., Sayers, E.W.: GenBank. - Nucl. Acids Res. 39 (Suppl.): D32-D37, 2011.

Bradford, M.M.: A rapid and sensitive method for the quantitation of microgram quantities of protein utilizing the principle of protein-dye binding. - Anal Biochem. 72: 248254, 1976.

Broin, M., Cuine, S., Eymery, F., Rey, P.: The plastidic 2cysteine peroxiredoxin is a target for a thioredoxin involved in the protection of the photosynthetic apparatus against oxidative damage. - Plant Cell 14: 1417-1432, 2002.

Carvalho, L.C., Vilela, B.J., Mullineaux, P.M., Amâncio, S.: Comparative transcriptomic profiling of Vitis vinifera under high light using a custom-made array and the Affymetrix gene chip. - Mol. Plant 4: 1038-1051, 2011.

Carvalho, L.C., Vilela, B.J., Vidigal, P., Mullineaux, P.M., Amâncio, S.: Activation of the ascorbate-glutathione cycle is an early response of micropropagated Vitis vinifera L. explants transferred to ex vitro. - Int. J. Plant Sci. 167: 759770, 2006.

Coito, J.L., Rocheta, M., Carvalho, L.C., Amâncio, S.: Microarray-based uncovering reference genes for quantitative real time PCR in grapevine under abiotic stress. - BMC Res. Notes 5: 220, 2012.

Dietz, K.J.: The dual function of plant peroxiredoxins in antioxidant defence and redox signaling. - Subcell. Biochem. 44: 267-294, 2007.

Dietz, K.J.: Peroxiredoxins in plants and cyanobacteria. Antioxid. Redox Signal 15: 1129-1159, 2011.

Dietz, K.J., Horling, F., Konig, J., Baier, M.: The function of the chloroplast 2-cysteine peroxiredoxin in peroxide detoxification and its regulation. - J. exp. Bot. 53: 13211329, 2002.

Dietz, K.J., Jacob, S., Oelze, M.L., Laxa, M., Tognetti, V., De Miranda, S.M., Baier, M., Finkemeier, I.: The function of peroxiredoxins in plant organelle redox metabolism. - J. exp. Bot. 57: 1697-1709, 2006.

Felsenstein, J.: Confidence limits on phylogenies: an approach using the bootstrap. - Evolution 39: 783-791, 1985.

Gama, F., Keech, O., Eymery, F.O., Finkemeier, I., Gelhaye, E., Gardestrom, P., Dietz, K.J., Rey, P., Jacquot, J.P.,
Rouhier, N.: The mitochondrial type II peroxiredoxin from poplar. - Physiol. Plant. 129: 196-206, 2007.

Gasteiger, E., Hoogland, C., Gattiker, A., Duvaud, S., Wilkins, M.R., Appel, R.D., Bairoch, A.: Protein identification and analysis tools on the ExPASy server. - In: Walker, J.M. (ed.): The Proteomics Protocols Handbook. Pp. 571-607. Humana Press, Totowa 2005.

Geuna, F., Hartings, H., Scienza, A.: A new method for rapid extraction of high quality RNA from recalcitrant tissues of grapevine. - Plant mol. Biol. Rep. 16: 61-67, 1998.

Haslekas, C., Stacy, R.A., Nygaard, V., Culianez-Macia, F.A., Aalen, R.B.: The expression of a peroxiredoxin antioxidant gene, AtPerl, in Arabidopsis thaliana is seed-specific and related to dormancy. - Plant mol. Biol. 36: 833-845, 1998.

Haslekas, C., Viken, M.K., Grini, P.E., Nygaard, V., Nordgard, S.H., Meza, T.J., Aalen, R.B.: Seed 1-cysteine peroxiredoxin antioxidants are not involved in dormancy, but contribute to inhibition of germination during stress. Plant Physiol. 133: 1148-1157: 2003.

Horling, F., Konig, J., Dietz, K.J.: Type II peroxiredoxin C, a member of the peroxiredoxin family of Arabidopsis thaliana: its expression and activity in comparison with other peroxiredoxins. - Plant Physiol. Biochem. 40: 491499, 2002.

Horling, F., Lamkemeyer, P., Konig, J., Finkemeier, I., Kandlbinder, A., Baier, M., Dietz, K.J.: Divergent light-, ascorbate-, and oxidative stress-dependent regulation of expression of the peroxiredoxin gene family in Arabidopsis. - Plant Physiol. 131: 317-325, 2003.

Horton, P., Park, K.J., Obayashi, T., Fujita, N., Harada, H., Adams-Collier, C.J., Nakai, K.: WoLFPSORT: protein localization predictor. - Nucl. Acids Res. 35 (Suppl.): W585-W587, 2007.

Hossain, M.A., Fujita, M.: Regulatory role of components of ascorbate-glutathione (AsA-GSH) pathway in plant tolerance to oxidative stress. - In: Anjum, N.A., Umar, S., Ahmed, A. (ed.): Oxidative Stress in Plants: Causes, Consequences and Tolerance. Pp. 81-147. IK International Publishing House, New Delhi 2011.

Huang, L., McCluskey, M.P., Ni, H., LaRossa, R.A.: Global gene expression profiles of the cyanobacterium Synechocystis sp. strain PCC 6803 in response to irradiation with UV-B and white light. - J. Bacteriol. 184: 6845-6858, 2002.

Jing, L.W., Chen, S.H., Guo, X.L., Zhang, H., Zhao, Y.X.: Overexpression of a chloroplast-located peroxiredoxin $\mathrm{Q}$ gene, $S s \operatorname{Pr} Q Q$, increases the salt and low-temperature tolerance of Arabidopsis. - J. Integr. Plant Biol. 48: 12441249, 2006

Konig, J., Baier, M., Horling, F., Kahmann, U., Harris, G., Schurmann, P., Dietz, K.J.: The plant-specific function of 2-Cys peroxiredoxin-mediated detoxification of peroxides in the redox-hierarchy of photosynthetic electron flux. Proc. nat. Acad. Sci. USA 99: 5738-5743, 2002.

Lamkemeyer, P., Laxa, M., Collin, V., Li, W., Finkemeier, I., Schottler, M.A., Holtkamp, V., Tognetti, V.B., IssakidisBourguet, E., Kandlbinder, A., Weis, E., Miginiac-Maslow, M., Dietz, K.J.: Peroxiredoxin Q of Arabidopsis thaliana is attached to the thylakoids and functions in context of photosynthesis. - Plant J. 45: 968-981, 2006.

Larkin, M.A., Blackshields, G., Brown, N.P., Chenna, R., McGettigan, P.A., McWilliam, H., Valentin, F., Wallace, I.M., Wilm, A., Lopez, R., Thompson, J.D., Gibson, T.J., 
Higgins, D.G.: Clustal W and Clustal X version 2.0. Bioinformatics 23: 2947-2948, 2007.

Li, H., Singh, A.K., McIntyre, L.M., Sherman, L.A.: Differential gene expression in response to hydrogen peroxide and the putative PerR regulon of Synechocystis sp. strain PCC 6803. - J. Bacteriol. 186: 3331-3345, 2004.

Marchler-Bauer, A., Lu, S., Anderson, J.B., Chitsaz, F., Derbyshire, M.K., DeWeese-Scott, C., Fong, J.H., Geer, L.Y., Geer, R.C., Gonzales, N.R., Gwadz, M., Hurwitz, D.I., Jackson, J.D., Ke, Z., Lanczycki, C.J., Lu, F., Marchler, G.H., Mullokandov, M., Omelchenko, M.V., Robertson, C.L., Song, J.S., Thanki, N., Yamashita, R.A., Zhang, D., Zhang, N., Zheng, C., Bryant, S.H.: CDD: a conserved domain database for the functional annotation of proteins. - Nucl. Acids Res. 39 (Suppl.): D225-D229, 2011.

Mittler, R.: Oxidative stress, antioxidants, and stress tolerance. Trends Plant Sci. 7: 405-410, 2002.

Mowla, S.B., Thomson, J.A., Farrant, J.M., Mundree, S.G.: A novel stress-inducible antioxidant enzyme identified from the resurrection plant Xerophyta viscosa Baker. - Planta 215: 716-726, 2002.

Parsonage, D., Karplus, P.A., Poole, L.B.: Substrate specificity and redox potential of $\mathrm{AhpC}$, a bacterial peroxiredoxin. Proc. nat. Acad. Sci. USA 105: 8209-8214, 2008.

Perez-Perez, M.E., Mata-Cabana, A., Sanchez-Riego, A.M., Lindahl, M., Florencio, F.J.: A comprehensive analysis of the peroxiredoxin reduction system in the cyanobacterium Synechocystis sp. strain PCC 6803 reveals that all five peroxiredoxins are thioredoxin dependent. - J. Bacteriol. 191: 7477-7489, 2009.

Petersson, U.A., Kieselbach, T., Garcia-Cerdan, J.G., Schroder, W.P.: The Prx Q protein of Arabidopsis thaliana is a member of the luminal chloroplast proteome. - FEBS Lett. 580: 6055-6061, 2006.

Pulido, P., Cazalis, R., Cejudo, F.J.: An antioxidant redox system in the nucleus of wheat seed cells suffering oxidative stress. - Plant J. 57: 132-145, 2009.

Pulido, P., Spinola, M.C., Kirchsteiger, K., Guinea, M., Pascual, M.B., Sahrawy, M., Sandalio, L.M., Dietz, K.J., Gonzalez, M., Cejudo, F.J.: Functional analysis of the pathways for 2-Cys peroxiredoxin reduction in Arabidopsis thaliana chloroplasts. - J. exp. Bot. 61: 4043-4054, 2010.

Rhee, S.G., Kang, S.W., Jeong, W., Chang, T.S., Yang, K.S., Woo, H.A.: Intracellular messenger function of hydrogen peroxide and its regulation by peroxiredoxins. - Curr. Opin. Cell Biol. 17: 183-189, 2005.

Rhue R.D., Grogan C.O., Stockmeyer E.W., Evert H.L.: Genetic control of aluminium tolerance in corn. - Crop Sci. 18: 1063-1067, 1978.
Romero-Puertas, M.C., Laxa, M., Matte, A., Zaninotto, F., Finkemeier, I., Jones, A.M., Perazzolli, M., Vandelle, E., Dietz, K.J., Delledonne, M.: S-nitrosylation of peroxiredoxin II E promotes peroxynitrite-mediated tyrosine nitration. - Plant Cell 19: 4120-4130, 2007.

Rouhier, N., Jacquot, J.P.: The plant multigenic family of thiol peroxidases. - Free Rad. Biol. Med. 38: 1413-1421, 2005.

Saitou, N., Nei, M.: The neighbor-joining method: a new method for reconstructing phylogenetic trees. - Mol. Biol. Evol. 4: 406-425, 1987.

Schwarz, R., Dayhoff, M.: Matrices for detecting distant relationships. - In: Dayhoff, M. (ed.): Atlas of Protein sequences. Pp. 353-358. Nat. Biomed. Res. Found., Washington 1979.

Song, Y., Lin, Y., Tong, S., Hou, H.: Molecular cloning, promoter analysis, and expression profile of $V v E R F 3 b$ gene in Vitis vinifera. - Biol. Plant. 56: 31-36, 2012.

Sweetlove, L.J., Heazlewood, J.L., Herald, V., Holtzapffel, R., Day, D.A., Leaver, C.J., Millar, A.H.: The impact of oxidative stress on Arabidopsis mitochondria. - Plant J. 32: 891-904, 2002.

Tamura, K., Dudley, J., Nei, M., Kumar, S.: MEGA4: molecular evolutionary genetics analysis (MEGA) software version 4.0. - Mol. Biol. Evol. 24: 1596-1599, 2007.

Tripathi, B.N., Bhatt, I., Dietz, K.J.: Peroxiredoxins: a less studied component of hydrogen peroxide detoxification in photosynthetic organisms. - Protoplasma 235: 3-15, 2009.

Upadhyay, A., Upadhyay, A.K., Bhirangi, R.A.: Expression of $\mathrm{Na}^{+} / \mathrm{H}^{+}$antiporter gene in response to water and salinity stress in grapevine rootstocks. - Biol. Plant. 56: 762-766, 2012.

Vandenabeele, S., Vanderauwera, S., Vuylsteke, M., Rombauts, S., Langebartels, C., Seidlitz, H.K., Zabeau, M., Van Montagu, M., Inzé, D., Van Breusegem, F.: Catalase deficiency drastically affects gene expression induced by high light in Arabidopsis thaliana. - Plant J. 39: 45-58, 2004.

Vilela, B.J., Carvalho, L.C., Ferreira, J., Amâncio, S.: Imaging of photooxidative stress symptoms and stomatal functioning in Vitis vinifera L. transferred from in vitro to ex vitro under increased light. - Plant Cell Rep. 26: 2149-2157, 2007.

Wang, L.J., Huang, W.D., Liu, Y.P., Zhan, J.C.: Changes in salicylic and abscisic acid contents during heat treatment and their effect on thermotolerance of grape plants. - Russ. J. Plant Physiol. 52: 516-520, 2005.

Wood Z.A., Schroder E., Harris J.R., Poole L.B.: Structure, mechanism and regulation of peroxiredoxins. - Trends Biochem. Sci. 28: 32-40, 2003. 